\title{
Computational technique for simulating variable-order fractional Heston model with application in US stock market
}

\author{
Zeinab Salamat Mostaghim $^{1} \cdot$ Behrouz Parsa Moghaddam $^{1} \mathbb{D} \cdot$ Hossein Samimi Haghgozar $^{2}$
}

Received: 5 July 2018 / Accepted: 12 October 2018 / Published online: 22 October 2018

(c) The Author(s) 2018

\begin{abstract}
In this paper, a numerical technique is developed to discretize variable-order fractional Heston differential equation. The proposed strategy is followed by an optimization technology, genetic algorithm, for tuning the unknown parameters in the proposed model. The performance of the model is analyzed to profit and loss 500 close index from the US stock markets. Simulations illustrate the application of the proposed technique.
\end{abstract}

Keywords Fractional calculus $\cdot$ Stochastic calculus $\cdot$ Computational techniques $\cdot$ Optimization $\cdot$ Variable-order fractional Heston model $\cdot$ Stock price

Mathematics Subject Classification $26 \mathrm{~A} 33 \cdot 34 \mathrm{~A} 08 \cdot 60 \mathrm{H} 35 \cdot 93 \mathrm{~B} 40 \cdot 49 \mathrm{~J} 15$

\section{Introduction}

The Black-Scholes model has been introduced based on the geometric Brownian motion to providing a closed-form pricing formula for the European options and describing the behavior of underlying asset prices [1-3]. The assumptions of the Black-Scholes model are unrealistic due to its inability to generate volatility satisfying the market observations, being nonnegative and mean-reverting [4-6]. To overcome this limitation, in 1993, Heston suggested Heston's stochastic volatility (HSV) model [6]. The HSV model has been extended in finance for modeling the dynamics of implied volatilities and providing their user with simple breakeven accounting conditions for the profit and loss (S\&P) of a hedged position [2, 7-17].

Behrouz Parsa Moghaddam

parsa@liau.ac.ir

Zeinab Salamat Mostaghim

salamatmostaghim93@liau.ac.ir

Hossein Samimi Haghgozar

samimi@guilan.ac.ir

1 Department of Mathematics, Lahijan Branch, Islamic Azad University, Lahijan, Iran

2 Department of Statistics, Faculty of Mathematical Sciences, University of Guilan, Rasht, Iran
The HSV model, in the risk-neutral measure, is presented as follows

$$
\begin{aligned}
& \left(\begin{array}{l}
\mathscr{D}_{t} S(t) \\
\mathscr{D}_{t} V(t)
\end{array}\right)=\left(\begin{array}{c}
r S(t) \\
\kappa(\theta-V(t))
\end{array}\right) \\
& \quad+\left(\begin{array}{cc}
\sqrt{V(t)} S(t) & 0 \\
\rho \sigma \sqrt{V(t)} & \sigma \sqrt{V(t)\left(1-\rho^{2}\right)}
\end{array}\right)\left(\begin{array}{c}
\frac{\mathrm{d} \omega_{1}}{\mathrm{~d} t} \\
\frac{\mathrm{d} \omega_{2}}{\mathrm{~d} t}
\end{array}\right),
\end{aligned}
$$

where $\mathscr{D}_{t}[\cdot] \equiv \frac{\mathrm{d}}{\mathrm{d} t}[\cdot]$ is the integer-order derivative operator, $S(t)$ denotes the stock price, and $V(t)$ denotes it's return variance at time $t$ that referred to in the literature as volatility [18]. The parameters $r$ and $\theta$ show the risk-free rate and the volatility long-run mean, respectively. Moreover, the parameter $\kappa$ reveals the mean reversion rate toward $\theta$ that controls the speed volatility going back to its average value. The volatility of volatility, $\sigma$, is a significant risk factor that depicts the kurtosis in the stock return rate distribution. The Brownian motions, $\omega_{1}(t)$ and $\omega_{2}(t)$ with correlation coefficient, $\rho \in[-1,1]$, are in the risk-neutral measure.

Despite its popularity, research on efficient discretizations of the continuous time dynamics of the Heston model has generated less attention by researchers. In 2006, an exact simulation technique was suggested by Broadie and Kaya for the HSV model [19]. Subsequently, in [20], a simulation scheme including the quadratic-exponential technique has been developed, and in [21], a second-order 
discretization scheme has been discussed. Along the same line of thought, the drift-implicit Milstein algorithm for the volatility [22], a Euler discretization for the log-Heston price [16] and an analytic approach for degenerate parabolic problem associated with the HSV model [17] were presented.

In the last decades, the theory of fractional calculus was motivated as a useful mathematical tool to handle application of associated concepts in the areas of physics, chemistry, economics, finance and engineering sciences [23-34]. To the author's best knowledge, while numerous stochastic volatility models driven by fractional Brownian motion have been considered [35-38], stochastic volatility models with fixed-order and variable-order fractional derivative operators have not been carried out and this topic is far from being fully explored. More recently, just a numerical discretization technique has been proposed for approximation of a class of fractional stochastic differential equations driven by Brownian motion [39].

In this work, we assume that Brownian motions, $\omega_{1}(t)$ and $\omega_{2}(t), t \geq 0$, be real continuous functions that defined on a filtered probability space $(\Omega, \mathcal{F}, \mathbb{P})$ with a normal filtration $\left(\mathcal{F}_{t}\right)_{t \geq 0}$. We consider the variable-order fractional HSV (VOF-HSV) model as

$$
\begin{aligned}
& \left(\begin{array}{cc}
v o D_{t}^{\gamma(\cdot)} S(t) \\
0 \\
v o \mathscr{D}_{t}^{\beta(\cdot)} V(t)
\end{array}\right)=\left(\begin{array}{c}
r S(t) \\
\kappa(\theta-V(t))
\end{array}\right) \\
& \quad+\left(\begin{array}{cc}
\sqrt{V(t)} S(t) & 0 \\
\rho \sigma \sqrt{V(t)} & \sigma \sqrt{V(t)\left(1-\rho^{2}\right)}
\end{array}\right)\left(\begin{array}{c}
\frac{\mathrm{d} \omega_{1}}{\mathrm{~d} t} \\
\frac{\mathrm{d} \omega_{2}}{\mathrm{~d} t}
\end{array}\right),
\end{aligned}
$$

where $\frac{1}{2}<\gamma(t), \beta(t) \leq 1$. The symbols ${ }_{0}^{\nu o} \mathscr{D}_{t}^{\gamma(\cdot)}[\cdot]$ and ${ }_{0}^{v o} \mathscr{D}_{t}^{\beta(\cdot)}[\cdot]$ are the fractional derivative operators of variable-order $\gamma(t) \in \mathbb{R}^{+}$and $\beta(t) \in \mathbb{R}^{+}$, respectively. In general, variableorder fractional derivative operator was defined by Lorenzo and Hartley as [40]

${ }_{0}^{v o} \mathscr{D}_{t}^{\gamma(\cdot)} u(t)=\frac{1}{\Gamma(m-\gamma(t))} \int_{0}^{t} \frac{u^{(m)}(\zeta) \mathrm{d} \zeta}{(t-\zeta)^{\gamma(t)+1-m}} \quad m-1<\gamma(\cdot) \leq m$,

where $u(t)$ is $(m-1), m \in \mathbb{N}$, times continuously differentiable and $u^{(m)}(t)$ is once integrable, $\zeta$ is an auxiliary variable that belongs to the interval $[0, t]$, and $\Gamma(\cdot)$ denotes the Gamma function. The concept and properties of variableorder fractional derivative operators and their application have been discussed in [41-45].

The outline of the paper is organized as follows. In "Optimal VOF-HSV model" section, the tuning technique is described for designing optimal VOF-HSV model for US stock market. For this propose, a discretization algorithm for the VOF-HSV model is proposed and followed by an optimization technique, genetic algorithm (GA), for tuning the unknown parameters in the VOF-HSV model. The advantages of using the VOFHSV model are shown and verified by considering different performance criteria in "Numerical results" section. Finally, "Conclusion" section draws the conclusions.

\section{Optimal VOF-HSV model}

Throughout this paper, we let $\Lambda=[0, T]$ with a uniform grid $t_{j}=j h, j=0,1, \ldots, n, n \in \mathbb{Z}^{+}$, such that $n+1=\frac{T}{h}$, and $\gamma_{n}=\gamma\left(t_{n}\right), \beta_{n}=\beta\left(t_{n}\right), S_{n}=S\left(t_{n}\right)$ and $V_{n}=V\left(t_{n}\right)$. In this section, an optimization strategy is formulated based on discretizing the equations in (2) and minimizing performance index under the following propositions.

Proposition 2.1 [46] The discretized expression of variableorder fractional derivative ${ }_{0}^{\text {vo }} \mathscr{D}_{t}^{\gamma(\cdot)}[\cdot]$ in (3) is obtained by the forward finite-difference approximation (M-algorithm) as

${ }_{0}^{v o} \mathscr{D}_{t_{n+1}}^{\gamma(\cdot)} u(t)=\frac{h^{-\gamma_{n+1}}}{\Gamma\left(m+1-\gamma_{n+1}\right)} \sum_{j=0}^{n} \psi_{m, n, j} \Delta_{h}^{m} u_{n-j}+\mathcal{O}\left(h^{m+1-\gamma_{n}}\right)$,

where $h$ denotes the uniform step size, $\psi_{m, n, j}=\left[(j+1)^{m-\gamma_{n}}-j^{m-\gamma_{n}}\right]$,

$\Delta_{h}^{m} u(t)=\sum_{i=0}^{m}(-1)^{i}\left(\begin{array}{c}m \\ i\end{array}\right) u(t+(m-i) h)$

where $\mathcal{O}(\cdot)$ denotes convergence order of approximation.

Proposition 2.2 Let $0<\gamma(t) \leq 1$. Assume that $u(t)$ be a function in $\mathbb{L}^{2}\left(\Omega, \mathcal{F}_{t}, \mathbb{P}\right)$ and for every subinterval $t \in\left[t_{j}, t_{j+1}\right] \subseteq \Lambda, u(t) \in C^{2}\left[t_{j}, t_{j+1}\right]$ and $\left\|u^{\prime \prime}(t)\right\| \leq \rho$, $j=0,1, \ldots, n$. Then, there exists an $\gamma_{n+1}$ dependent constant, i.e., $C_{\gamma_{n+1}}>0$, such that the truncated error of the variable-order fractional derivative operator obtained by the M-algorithm satisfies

$$
\begin{aligned}
& \mathbb{E}\left[\left|{ }_{0}^{v o} \mathscr{D}_{t_{n+1}}^{\gamma(\cdot)} u(t)-\left({ }_{0}^{v o} \mathscr{D}_{t_{n+1}}^{\gamma(\cdot)} u(t)\right)_{M \text {-algorithm }}\right|\right] \\
& \leq C_{\gamma_{n+1}} h^{2-\gamma_{n+1}}=\mathcal{O}\left(h^{2-\gamma_{n+1}}\right) \text {, }
\end{aligned}
$$

where $C_{\gamma_{n+1}}=\frac{(n+1)^{1-\gamma_{n+1}} \varrho}{2 \Gamma\left(2-\gamma_{n+1}\right)}$.

Proof Assume that $u_{j}$ be an approximation of $u_{j}(t)$ in the subinterval $\left[t_{j}, t_{j+1}\right] \subseteq\left[0, t_{n+1}\right)=[0, T), j=0,1, \ldots, n$ and

$\mathscr{E}_{j}(t)=u_{j}^{\prime}-u_{j}^{\prime}(t)=\frac{h}{2} u_{j}^{\prime \prime}(t)$.

Let $\mathscr{E}(t)$ be the error function in the interval $\left(0, t_{n}\right]$; therefore, 


$$
\begin{aligned}
& \mathbb{E}\left[\left|{ }_{0}^{v o} \mathscr{D}_{t_{n+1}^{\gamma(\cdot)}}^{\gamma(t)}-\left({ }_{0}^{v o} \mathscr{D}_{t_{n+1}}^{\gamma(\cdot)} u(t)\right)_{M \text {-algorithm }}\right|\right] \\
& =\frac{1}{\Gamma\left(1-\gamma_{n+1}\right)} \mathbb{E}\left[\sum_{j=0}^{n} \int_{t_{j}}^{t_{j+1}}\left\|\frac{\mathscr{C}_{j}(\zeta)}{\left(t_{n+1}-\zeta\right)^{\gamma_{n+1}}}\right\| d \zeta\right] \\
& =\frac{1}{\Gamma\left(1-\gamma_{n+1}\right)} \mathbb{E}\left[\sum_{j=0}^{n} \int_{t_{j}}^{t_{j+1}}\left\|\frac{h u_{j}^{\prime \prime}(t)}{2\left(t_{n+1}-\zeta\right)^{\gamma_{n+1}}}\right\| d \zeta\right] \\
& \leq \frac{h t_{n+1}^{1-\gamma_{n+1}} \varrho}{2 \Gamma\left(2-\gamma_{n+1}\right)} \\
& =\frac{(n+1)^{1-\gamma_{n+1}} \rho h^{2-\gamma_{n+1}}}{2 \Gamma\left(2-\gamma_{n+1}\right)}=\mathcal{O}\left(h^{2-\gamma_{n+1}}\right) .
\end{aligned}
$$

Using Propositions 2.1, the discretized equations of VOFHSV model (2) are derived as follows

$$
\begin{aligned}
& \left(\begin{array}{c}
S_{n+1} \\
V_{n+1}
\end{array}\right)=\left(\begin{array}{c}
\left(r h^{\gamma_{n}} \Gamma\left(2-\gamma_{n}\right)+1\right) S_{n}-\Psi_{h, n, j} \\
\kappa h^{\beta_{n}} \Gamma\left(2-\beta_{n}\right)\left(\theta-V_{n}\right)+V_{n}-\Phi_{h, n, j}
\end{array}\right) \\
& +\left(\begin{array}{cc}
h^{\gamma_{n}-1} \Gamma\left(2-\gamma_{n}\right) \sqrt{V_{n}} S_{n} & 0 \\
\rho \sigma h^{\beta_{n}-1} \Gamma\left(2-\beta_{n}\right) \sqrt{V_{n}} & \sigma h^{\beta_{n}-1} \Gamma\left(2-\beta_{n}\right) \sqrt{V_{n}\left(1-\rho^{2}\right)}
\end{array}\right)\left(\begin{array}{c}
\Delta_{h} \omega_{1, n} \\
\Delta_{h} \omega_{2, n}
\end{array}\right) .
\end{aligned}
$$

where $\Psi_{h, n, j}=\sum_{j=1}^{n} \psi_{1, n, j} \Delta_{h} S_{n-j}$ and $\Phi_{h, n, j}=\sum_{j=1}^{n} \psi_{1, n, j} \Delta_{h} V_{n-j}$

There are different performance criteria for measuring goodness of fit typically summarizing discrepancy between observed values and estimated values by the prediction model. We analyze the performance of proposed models, in the viewpoint of root-mean-square error (RMSE) or the residual standard deviation defined as

$\mathrm{RMSE}=\frac{\mathrm{RSS}}{\sqrt{N-p-1}}$,

where RSS denotes the square root of residual sum of squares, $N$ and $p$ are the number of observations and parameters in the equation, respectively [47].

For estimating the Bayesian information criterion (BIC), we apply the following formula [47]

$\mathrm{BIC}=N \times \ln \left(\frac{\mathrm{RSS}}{N}\right)+2 \ln (N)$

Moreover, the relative quality of statistical models for a given set of data can be analyzed in the perspective of the Akaike information criterion (AIC) defined as [48]

$\mathrm{AIC}=N \times \ln (\mathrm{RSS})+2 p$.

A lower RMSE, BIC or AIC value indicates a better fit.

\section{Numerical results}

In this section, fixed-order and VOF-HSV models are used to estimate the behavior of the price of S\&P 500 index of American options. The S\&P 500 is a US stock market index that covers all the large market public companies recorded on the New York Stock Exchange (NYSE) or National Association of Securities Dealers Automated Quotations (NASDAQ) [49]. For practical applicability, in this work, the parameters of the financial market model are chosen based on [50]. In [50], authors obtained estimate values of parameters for the typical Heston model by considering S\&P 500 index returns. Throughout the numerical analysis, the main parameters satisfy that the mean reversion rate converges to $\kappa=2.75$, the long-run mean of the volatility converges to $\theta=0.035$, the volatility of volatility converges to $\sigma=0.425$, and the correlation coefficient converges to $\rho=-0.4644$.

For constructing VOF-HSV model, $\gamma(t)=c_{1}+c_{2} t$ and $\beta(t)=c_{3}+c_{4} t$ functions where $0 \leq c_{i} \leq 1, i=1, \ldots, 4$, with four unknown parameters being considered. Next, an optimization method, GA, is used to find the optimal values of the unknowns $c_{i}, i=1, \ldots, 4$, by minimizing the mean absolute error (MAE) at the discretized points goodness, that is, by minimizing MAE $=\frac{1}{N} \sum_{n=1}^{N}\left|S\left(t_{n}\right)-\hat{S}_{n}\right|$, where $S\left(t_{n}\right)$ and $\hat{S}_{n}$ are the discretized value of the model and experimental value, respectively. The optimization algorithm is based on discretizing (6) by using the $M$-algorithm formulated in Propositions 2.1 with $n=1227$ equal mesh points, corresponding to observed data, so that approximation error can be determined by using Proposition 2.2. All the computations are performed under Maple v18 on an Intel (R) Core (TM) i7-7500U CPU @ 2.70 GHz machine.

Table 1 shows the obtained values for the unknown parameters of the integer-order (i.e., $c_{1}=c_{3}=1$ and $c_{2}=c_{4}=0$ ), the fixed-order fractional (i.e., $c_{2}=c_{4}=0$ ), and the VOF-HSV models when $t \leq 1$. In Table 2 , the results in the perspective of the indices RMSE, AIC and BIC reveal that there is evidence of positive serial correlation in all considered cases of HSV models and the variable-order fractional model gives the best fitting out of the all proposed models. Moreover, there is a significant improvement in the response estimation by using the fractional

Table 1 The MAEs and optimal parameters of the integer-order, the fixed-order fractional and VOF-HSV models for $\gamma(t)=c_{1}+c_{2} t$ and $\beta(t)=c_{3}+c_{4} t$ in the interval $[0,1]$

\begin{tabular}{llllll}
\hline HSV models & MAE & $c_{1}$ & $c_{2}$ & $c_{3}$ & $c_{4}$ \\
\hline Integer-order & 69.43743 & 1 & 0 & 1 & 0 \\
$\begin{array}{l}\text { Fixed-oder fractional } \\
\begin{array}{l}\text { Variable-order frac- } \\
\text { tional }\end{array}\end{array}$ & 44.95400 & 0.8960 & 0 & 0.9292 & 0 \\
\hline
\end{tabular}


Table 2 Comparison of RMSE, AIC and BIC of integer-order and optimal fixed- and VOF-HSV models with $n=1227$ equal mesh points in the interval $[0,1]$

\begin{tabular}{llll}
\hline HSV models & RMSE & AIC & BIC \\
\hline Integer-order & $2.9030 \times 10^{5}$ & 19787.83 & 11094.67 \\
Fixed-oder fractional & $1.3501 \times 10^{5}$ & 18849.18 & 10156.02 \\
Variable-order fractional & $1.1700 \times 10^{5}$ & 18673.73 & 9980.580 \\
\hline
\end{tabular}

HSV models. Figures 1 and 2 depict the numerical simulations of stock price, $S(t)$, and it's return variance, $V(t)$, with integer-order (i.e., $\gamma(t)=\beta(t)=1$ ), the fixed-order fractional (i.e., $\gamma(t)=0.8960$ and $\beta(t)=0.9292$ ), and the variable-order fractional (i.e., $\gamma(t)=0.9010+0.0890 t$ and $\beta(t)=0.9088+0.0869 t)$ HSV models with $n=1227$ equal mesh points in the interval $[0,1]$.

Since proposed models cannot be solved analytically using the standard mathematical techniques, the upper $95 \%$ confidence interval (CI) was utilized to predict the behavior of sample trajectories of solutions by means of the approximated HSV models. Moreover, the statistical analysis of the process is provided in Table 3. In Table 3, the approximation values of the mean, median, first and third quartiles, kurtosis, skewness, standard deviation (STD) and 95\% CI of the 50 simulated trajectories in time for several variation of orders for stock prices are listed.

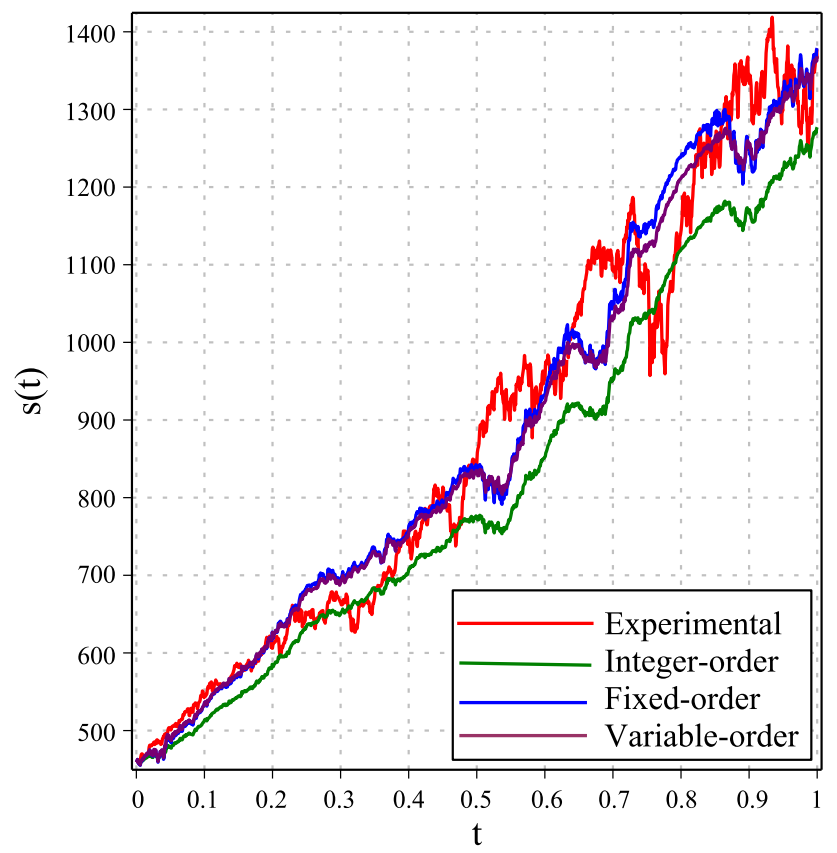

Fig. 1 The experimental stock price and the variation of the orders $\gamma(t)$ and $\beta(t)$ in the approximated HSV models for the experimental stock price tests

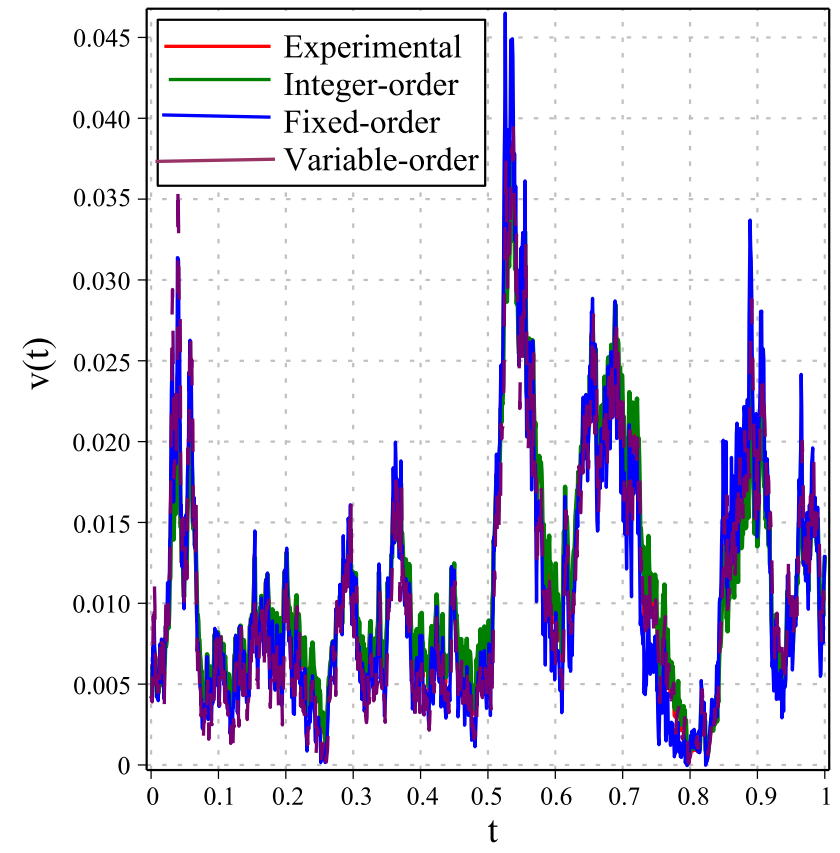

Fig. 2 The experimental stock price volatility and the variation of the orders $\gamma(t)$ and $\beta(t)$ in the approximated HSV models for the experimental volatility tests

Table 3 Approximation values of the mean, median, first and third quartiles, kurtosis, skewness, STD and 95\% CI of the 50 simulated trajectories for stock prices for various values of $\gamma(t)$ and $\beta(t)$, with $h=\frac{1}{1227}$ at $T=1$

\begin{tabular}{llll}
\hline $\begin{array}{l}\text { Statistical } \\
\text { indicators }\end{array}$ & HSV model & & \\
\cline { 2 - 4 } & Integer-order & $\begin{array}{l}\text { Fixed-order } \\
\text { fractional }\end{array}$ & $\begin{array}{l}\text { Variable-order } \\
\text { fractional }\end{array}$ \\
\hline Mean & 1279.50 & 1411.67 & 1367.36 \\
Median & 1306.74 & 1434.41 & 1386.61 \\
First quartile & 1136.37 & 1235.72 & 1171.84 \\
Third quartile & 1424.69 & 1596.35 & 1525.60 \\
Kurtosis & 2.630 & 2.640 & 2.811 \\
Skewness & -0.113 & -0.151 & -0.066 \\
STD & 203.02 & 263.55 & 237.48 \\
95\% CI & {$[881.57$,} & {$[895.12$,} & {$[901.90$,} \\
& $1677.42]$ & $1928.23]$ & $1832.82]$ \\
\hline
\end{tabular}

The comparison of experimental stock price and simulation results of 50 trajectories of the stock prices of proposed HSV models is shown in Fig. 3. In Fig. 3, the red circles indicate the trajectories corresponding to the observed stock price. The blue lines represent the simulation results of 50 trajectories of the approximated stock prices (Fig. 3, left column). The black and green lines indicate the $95 \% \mathrm{CI}$ area of the 50 trajectories and the empirical mean of the process (Fig. 3. right column), 
Fig. 3 The red circles indicate the trajectories corresponding to the observed stock price. The blue lines represent the simulation results of 50 trajectories of the approximated stock prices for proposed HSV models (left column). The black and the purple lines indicate the $95 \% \mathrm{CI}$ and first and third quartiles area of the 50 trajectories, respectively, and green line is the empirical mean of the process (right column), for proposed HSV models
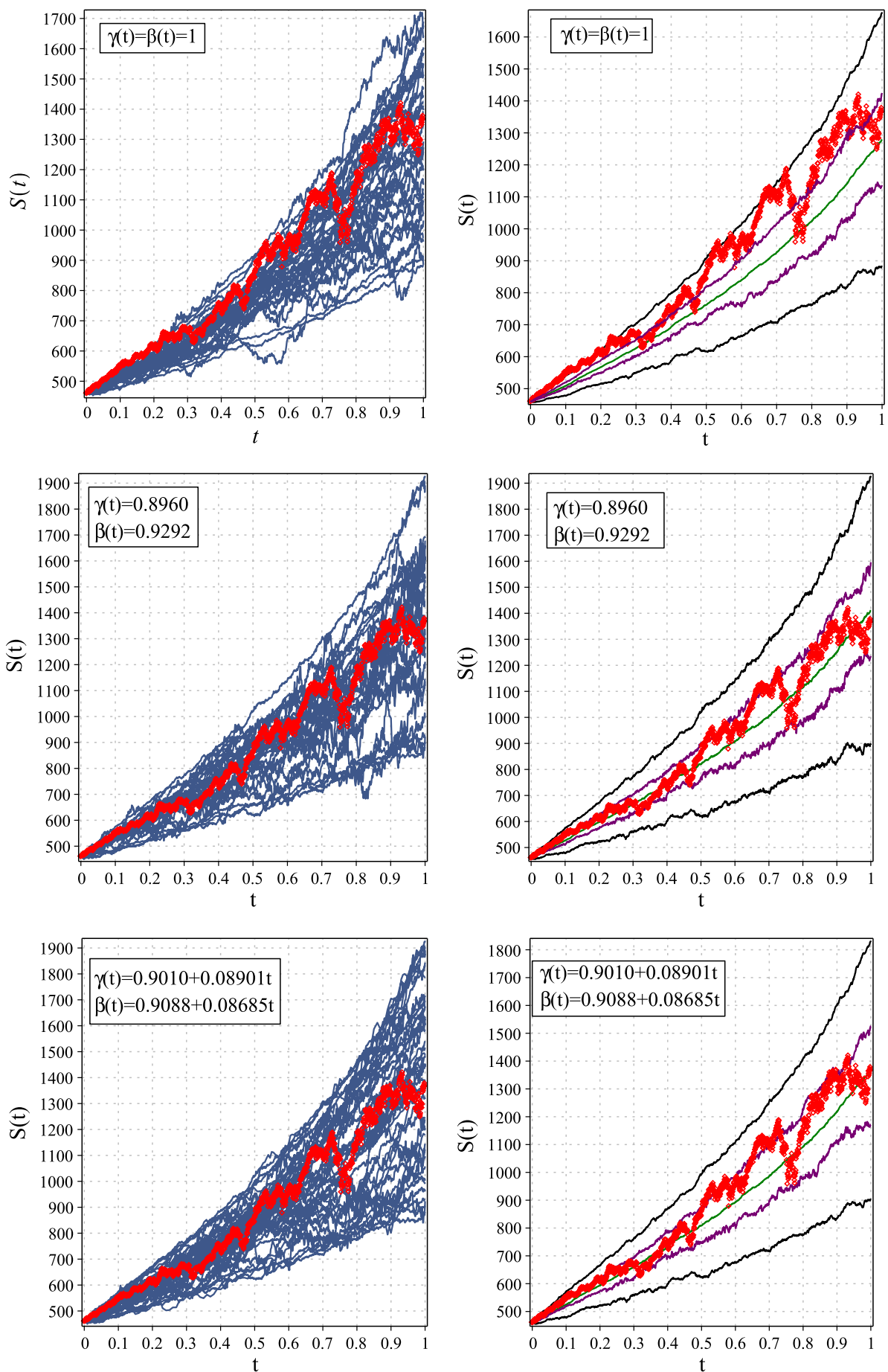

respectively, for proposed HSV models. The purple lines are first and third quartiles of these models.

\section{Conclusion}

It has been shown that using fixed-order and variable-order fractional derivative operators instead of an integer-order derivative operator in models can apparently lead to better results since one has an extra freedom degree. In this paper, the objective of introducing modified Heston model was to capture the complexities in simulating the scenarios of equity movement in finance. The proposed Heston's stochastic volatility models, due to random volatility and memory or hereditary properties, have more flexibility with market data than the constant volatility models. In this study, an optimization technique, genetic algorithm, was utilized to find an optimal set of unknown parameters of models. For this propose, an explicit numerical technique has been developed for solving 
Heston's stochastic volatility models. Furthermore, experimental stock price tests of a US stock market were used to estimate fixed-order and variable-order fractional Heston's stochastic volatility models. It was also shown that proposed fractional Heston's stochastic volatility models are significantly better in estimating the stock price in comparison to integer-order Heston's stochastic volatility models. Moreover, variable-order fractional Heston's stochastic volatility model is superior to fixed-order fractional Heston's stochastic volatility model.

Open Access This article is distributed under the terms of the Creative Commons Attribution 4.0 International License (http://creativeco mmons.org/licenses/by/4.0/), which permits unrestricted use, distribution, and reproduction in any medium, provided you give appropriate credit to the original author(s) and the source, provide a link to the Creative Commons license, and indicate if changes were made.

\section{References}

1. Black, F., Scholes, M.: The pricing of options and corporate liabilities. J. Polit. Econ. 81(3), 637-654 (1973). https://doi. org/10.1086/260062

2. Papi, M., Pontecorvi, L., Donatucci, C.: Weighted average price in the Heston stochastic volatility model. Decis. Econ. Finance 40(1-2), 351-373 (2017). https://doi.org/10.1007/ s10203-017-0197-5

3. Vajargah, K.F., Shoghi, M.: Simulation of stochastic differential equation of geometric Brownian motion by quasi- Monte Carlo method and its application in prediction of total index of stock market and value at risk. Math. Sci. 9(3), 115-125 (2015). https ://doi.org/10.1007/s40096-015-0158-5

4. Cox, J.C., Ingersoll, J.E., Ross, S.A.: A theory of the term structure of interest rates. Econometrica 53, 385-407 (1985)

5. Hull, J., White, A.: The pricing of options on assets with stochastic volatilities. J. Finance 42(2), 281-300 (1987). https:// doi.org/10.2307/2328253

6. Heston, S.L.: A closed-form solution for options with stochastic volatility with applications to bond and currency options. Rev. Financ. Stud. 6(2), 327-343 (1993). https://doi.org/10.1093/ $\mathrm{rfs} / 6.2 .327$

7. Ballestra, L.V., Pacelli, G., Zirilli, F.: A numerical method to price exotic path-dependent options on an underlying described by the Heston stochastic volatility model. J. Bank. Finance 31(11), 3420-3437 (2007). https://doi.org/10.1016/j.jbank fin.2007.04.013

8. Atiya, A.F., Wall, S.: An analytic approximation of the likelihood function for the Heston model volatility estimation problem. Quant. Finance 9(3), 289-296 (2009). https://doi. org/10.1080/14697680802595601

9. Hout, K.I., Foulon, S.: ADI finite difference schemes for option pricing in the Heston model with correlation. Int. J. Numer. Anal. Model. 7(2), 303-320 (2010)

10. Forde, M., Jacquier, A., Mijatović, A.: A note on essential smoothness in the Heston model. Finance Stoch. 15(4), 781-784 (2011). https://doi.org/10.1007/s00780-011-0162-z

11. Hout, K.I.: Finite difference approximation of hedging quantities in the Heston model. AIP (2012). https://doi. org/10.1063/1.4756108

12. Lenkšas, A., Mackevičius, V.: A second-order weak approximation of Heston model by discrete random variables. Lith.
Math. J. 55(4), 555-572 (2015). https://doi.org/10.1007/s1098 6-015-9298-4

13. Boguslavskaya, E., Muravey, D.: An explicit solution for optimal investment in Heston model. Theory Probab. Appl. 60(4), 679-688 (2016). https://doi.org/10.1137/s0040585x97t987946

14. Cui, Z., Feng, R., Anne, M.: Variable annuities with VIXlinked fee structure under a Heston-type stochastic volatility model. SSRN Electron. J. 21(3), 458-483 (2016). https://doi. org/10.2139/ssrn.2862657

15. Cui, Y., del Baño Rollin, S., Germano, G.: Full and fast calibration of the Heston stochastic volatility model. Eur. J. Oper. Res. 263(2), 625-638 (2017). https://doi.org/10.1016/j. ejor.2017.05.018

16. Altmayer, M., Neuenkirch, A.: Discretising the Heston model: an analysis of the weak convergence rate. IMA J. Numer. Anal. 37(4), 1930-1960 (2017). https://doi.org/10.1093/imanum/ drw063

17. Canale, A., Mininni, R.M., Rhandi, A.: Analytic approach to solve a degenerate parabolic PDE for the Heston model. Math. Methods Appl. Sci. 40(13), 4982-4992 (2017). https://doi. org/10.1002/mma.4363

18. Shreve, S.E.: Stochastic Calculus for Finance II: Continuoustime Models, vol. 11. Springer, New York (2004)

19. Broadie, M., Kaya, O.: Exact simulation of stochastic volatility and other affine jump diffusion processes. Oper. Res. 54(2), 217-231 (2006). https://doi.org/10.1287/opre.1050.0247

20. Andersen, L.: Simple and efficient simulation of the Heston stochastic volatility model. J. Comput. Finance 11(3), 1-42 (2008). https://doi.org/10.21314/jcf.2008.189

21. Alfonsi, A.: High order discretization schemes for the CIR process: application to affine term structure and Heston models. Math. Comput. 79(269), 209-209 (2010). https://doi. org/10.1090/s0025-5718-09-02252-2

22. Kahl, C., Gunther, M., Rossberg, T.: Structure preserving stochastic integration schemes in interest rate derivative modeling. Appl. Numer. Math. 58(3), 284-295 (2008). https://doi. org/10.1016/j.apnum.2006.11.013

23. Dabiri, A., Butcher, E.A.: Numerical solution of multi-order fractional differential equations with multiple delays via spectral collocation methods. Appl. Math. Model. 56, 424-448 (2018). https://doi.org/10.1016/j.apm.2017.12.012

24. Al-Khaled, K., Alquran, M.: An approximate solution for a fractional model of generalized Harry Dym equation. Math. Sci. 8(4), 125-130 (2014). https://doi.org/10.1007/s40096-015-0137-x

25. Arshed, S.: Quintic B-spline method for time-fractional superdiffusion fourth-order differential equation. Math. Sci. 11(1), 17-26 (2016). https://doi.org/10.1007/s40096-016-0200-2

26. Bhrawy, A.H., Zaky, M.A.: Numerical algorithm for the variable-order Caputo fractional functional differential equation. Nonlinear Dyn. 85(3), 1815-1823 (2016). https://doi. org/10.1007/s11071-016-2797-y

27. Li, X., Yang, X.: Error estimates of finite element methods for stochastic fractional differential equations. J. Comput. Math. 35(3), 346-362 (2017). https://doi.org/10.4208/jcm.1607-m2015-0329

28. Ahmadi, N., Vahidi, A.R., Allahviranloo, T.: An efficient approach based on radial basis functions for solving stochastic fractional differential equations. Math. Sci. 11(2), 113-118 (2017). https://doi.org/10.1007/s40096-017-0211-7

29. Zaky, M.A.: A Legendre spectral quadrature tau method for the multi-term time-fractional diffusion equations. Comput. Appl. Math. 37, 1-14 (2017). https://doi.org/10.1007/s4031 4-017-0530-1

30. Dabiri, A., Moghaddam, B.P., Machado, J.A.T.: Optimal variable-order fractional PID controllers for dynamical systems. J. 
Comput. Appl. Math. 339, 40-48 (2018). https://doi.org/10.1016/j. cam.2018.02.029

31. Keshi, F.K., Moghaddam, B.P., Aghili, A.: A numerical approach for solving a class of variable-order fractional functional integral equations. Comput. Appl. Math. 37(4), 4821-4834 (2018). https ://doi.org/10.1007/s40314-018-0604-8

32. Zaky, M.A., Doha, E.H., Taha, T.M., Baleanu, D.: New recursive approximations for variable-order fractional operators with applications. Math. Model. Anal. 23(2), 227-239 (2018). https://doi. org/10.3846/mma.2018.015

33. Machado, J.A.T., Moghaddam, B.P.: A robust algorithm for nonlinear variable-order fractional control systems with delay. Int. J. Nonlinear Sci. Numer. Simul. 19(3-4), 231-238 (2018). https:// doi.org/10.1515/ijnsns-2016-0094

34. Dabiri, A., Butcher, E.A., Nazari, M.: Coefficient of restitution in fractional viscoelastic compliant impacts using fractional Chebyshev collocation. J. Sound Vib. 388, 230-244 (2017). https://doi. org/10.1016/j.jsv.2016.10.013

35. Feng, X., Quan, S.: Pricing of option with power payoff driven by mixed fractional Brownian motion. In: 2010 3rd International Conference on Business Intelligence and Financial Engineering, IEEE, 2010, pp. 170-173. https://doi.org/10.1109/bife.2010.48

36. Ballestra, L.V., Pacelli, G., Radi, D.: A very efficient approach for pricing barrier options on an underlying described by the mixed fractional Brownian motion. Chaos Solitons Fractals 87, 240-248 (2016). https://doi.org/10.1016/j.chaos.2016.04.008

37. Bondarenko, V., Bondarenko, V., Truskovskyi, K.: Forecasting of time data with using fractional Brownian motion. Chaos Solitons Fractals 97, 44-50 (2017). https://doi.org/10.1016/j.chaos .2017 .01 .013

38. Panov, V.: Modern Problems of Stochastic Analysis and Statistics: Selected Contributions in Honor of Valentin Konakov, vol. 208. Springer, Berlin (2017)

39. Mostaghim, Z.S., Moghaddam, B.P., Haghgozar, H.S.: Numerical simulation of fractional-order dynamical systems in noisy environments. Comput. Appl. Math. 133, 1-15 (2018). https://doi. org/10.1007/s40314-018-0698-z

40. Lorenzo, C., Hartley, T.: Variable order and distributed order fractional operators. Nonlinear Dyn. 29(1-4), 57-98 (2002)

41. Zaky, M.A., Baleanu, D., Alzaidy, J.F., Hashemizadeh, E.: Operational matrix approach for solving the variable-order nonlinear Galilei invariant advection-diffusion equation. Adv. Differ. Equa. 2018(1), 102 (2018). https://doi.org/10.1186/s13662-018-1561-7
42. Moghaddam, B.P., Machado, J.A.T.: SM-algorithms for approximating the variable-order fractional derivative of high order. Fundam. Inf. 151(1-4), 293-311 (2017). https://doi.org/10.3233/ fi-2017-1493

43. Moghaddam, B.P., Machado, J.A.T.: A computational approach for the solution of a class of variable-order fractional integrodifferential equations with weakly singular kernels. Fract. Calc. Appl. Anal. 20(4), 1023-1042 (2017). https://doi.org/10.1515/ fca-2017-0053

44. Zaky, M.A.: A research note on the nonstandard finite difference method for solving variable-order fractional optimal control problems. J. Vib. Control 24(11), 2109-2111 (2018). https://doi. org/10.1177/1077546318761443

45. Moghaddam, B.P., Machado, J.A.T., Babaei, A.: A computationally efficient method for tempered fractional differential equations with application. Comput. Appl. Math. 37(3), 3657-3671 (2017). https://doi.org/10.1007/s40314-017-0522-1

46. Moghaddam, B.P., Machado, J.A.T.: Extended algorithms for approximating variable order fractional derivatives with applications. J. Sci. Comput. 71(3), 1351-1374 (2016). https://doi. org/10.1007/s10915-016-0343-1

47. Hossein-Zadeh, N.G.: Application of growth models to describe the lactation curves for test-day milk production in Holstein cows. J. Appl. Animal Res. 45(1), 145-151 (2016). https://doi. org/10.1080/09712119.2015.1124336

48. Guthery, F.S., Burnham, K.P., Anderson, D.R.: Model selection and multimodel inference: a practical information-theoretic approach. J. Wildl. Manag. 67(3), 655 (2003). https://doi. org/10.2307/3802723

49. Wang, X., He, X., Zhao, Y., Zuo, Z.: Parameter estimations of Heston model based on consistent extended Kalman filter. IFAC PapersOnLine 50(1), 14100-14105 (2017). https://doi. org/10.1016/j.ifacol.2017.08.1850

50. Zhang, J.E., Shu, J.: Pricing S\&P 500 index options with Heston's model. In: 2003 IEEE International Conference on Computational Intelligence for Financial Engineering, 2003. Proceedings., IEEE, pp. 85-92 (2003)

Publisher's Note Springer Nature remains neutral with regard to jurisdictional claims in published maps and institutional affiliations. 